\title{
Xenic Cultivation and Genotyping of Pathogenic Free-Living Amoeba from Public Water Supply Sources in Uganda
}

\author{
Celsus Sente, ${ }^{1}$ Joseph Erume, ${ }^{2}$ Irene Naigaga, ${ }^{1}$ Benigna Gabriela Namara, ${ }^{3}$ \\ Julius Mulindwa, ${ }^{2,4}$ Sylvester Ochwo, ${ }^{2}$ Phillip Kimuda Magambo, ${ }^{2}$ Charles Drago Kato, ${ }^{2}$ \\ Andrew Tamale, ${ }^{1}$ and Michael Ocaido ${ }^{1}$ \\ ${ }^{1}$ Department of Wildlife and Aquatic Animal Resources (WAAR), School of Veterinary Medicine and Animal Resources (SVAR), \\ College of Veterinary Medicine, Animal Resources and Biosecurity (COVAB), Makerere University, P.O. Box 7062, Kampala, Uganda \\ ${ }^{2}$ Department of Biomolecular Resources and Biolab Sciences, School of Biosecurity, Biotechnical and Laboratory Sciences (SBLS), \\ College of Veterinary Medicine, Animal Resources and Biosecurity (COVAB), Makerere University, P.O. Box 7062, Kampala, Uganda \\ ${ }^{3}$ Research Unit on AIDS, Medical Research Council (MRC)/Uganda Virus Research Institute (UVRI), P.O. Box 49, Entebbe, Uganda \\ ${ }^{4}$ Department of Biochemistry and Sports Science (BSS), College of Natural Sciences (CONAS), Kampala, Uganda
}

Correspondence should be addressed to Celsus Sente; csente37@gmail.com

Received 17 May 2016; Accepted 18 July 2016

Academic Editor: Xinhua Shu

Copyright (c) 2016 Celsus Sente et al. This is an open access article distributed under the Creative Commons Attribution License, which permits unrestricted use, distribution, and reproduction in any medium, provided the original work is properly cited.

Studies on waterborne parasites from natural environment and domestic water sources in Uganda are very scarce and unpublished. Water dwelling free-living amoebae (FLA) of the genus Acanthamoeba, Hartmannella, and Naegleria are often responsible for causing morbidities and mortalities in individuals with recent contact with contaminated water, but their presence in Uganda's public water supply sources is not known. We cultivated and genotyped FLA from natural and domestic water from Queen Elizabeth Protected Area (QEPA) and Kampala (KLA). The cultivated parasites were observed microscopically and recorded. The overall prevalence of FLA in QEPA (Acanthamoeba spp., 35\%; Hartmannella spp., 18.9\%; Naegleria spp., 13.5\%) and KLA (Acanthamoeba spp., 28.3\%; Naegleria spp., 16.6\%; Hartmannella spp., 23.1\%) were not significantly different. The highest prevalence across water sources in QEPA and KLA was observed for Acanthamoeba spp., followed by Hartmannella spp., and Naegleria spp. Overall FLA mean $( \pm \mathrm{SE})$ and mean $( \pm \mathrm{SE})$ across water sources were highest for Acanthamoeba spp. compared to other FLA but were not statistically significant $(p>0.05)$. Analysis of the FLA sequences produced 1 Cercomonas, 1 Nuclearia, 1 Bodomorpha, 2 Hartmannella, 5 Echinamoeba, and 7 Acanthamoeba partial sequences, indicating a muliplicity of water contaminants that need to be controlled by proper water treatment.

\section{Introduction}

Free-living amoeba (FLA) of the genera Naegleria, Acanthamoeba, and Hartmannella have been associated with water-related illnesses, especially in developed countries found in America, Europe, and the Middle East [1-4]. In many parts of Uganda, there is dependence on environmental water for washing, recreation, drinking, cooking, and other domestic purposes [5]. However, in most cases, this water is used without treatment, an act which predisposes Ugandans to FLA infection [5]. Since some of these amoebae are known to be fatal, it is imperative that their occurrence is monitored in order to assess the human and probably animal risks involved.

Studies worldwide have documented the common Naegleria, Acanthamoeba, and Hartmannella species associated with infectious diseases resulting from contaminated water sources utilised by communities. Considering the large numbers of Naegleria species, it is only N. fowleri, N. australiensis, and $N$. italica that are considered to be pathogenic [68] while other species such as $N$. gruberi, N. jadini, N. lovaniensis, $N$. indonesiensis, $N$. robinsoni, $N$. fultoni, and $N$. pagei have not yet been reported as pathogenic [8]. Information also denotes Acanthamoeba polyphaga, A. hatchetti, 
A. castellanii, A. culbertsoni, A. rhysodes, A. lugdunensis, A. quina, A. griffini, Balamuthia mandrillaris, Hartmannella vermiformis, and Vahlkampfia avara as pathogenic species mostly associated with keratitis in humans [9, 10]. Acanthamoeba spp. particularly renders amplification of Vibrio cholerae, Legionella pneumophila, Bacillus anthracis, and Mycoplasma tuberculosis inside their cells [10-12], a property of paramount importance to both humans and wildlife, in and around QEPA. Hartmannella spp. that have in many instances been isolated in mixed human amoebic keratitis infections are recently considered to be very pathogenic FLA [13]. This work, therefore, reports the different pathogenic FLA associated with tap and environmental surface water in QEPA and Kampala, an indicator towards water toxicity for the communities. In the past 10 years, outbreaks of cholera, typhoid, and other waterborne diseases have been documented in the study areas [14-17] and these could be linked to distribution by contaminated water sources as well.

The FLA of the genera Naegleria, Acanthamoeba, and Hartmannella predispose humans who collect water from various sources to diseases $[1,10,18]$. These pathogenic and opportunistic FLA are aerobic eukaryotic protists that occur worldwide and can potentially cause infections in humans and other animals $[10,19,20]$ The bacterial parasites Vibrio cholerae, Legionella pneumophila, Mycoplasma tuberculosis, and Bacillus anthracis are easily amplified within certain Acanthamoeba spp., Hartmannella spp., Naegleria spp., and other FLA consequently increasing their potential to cause cholera, legionellosis, tuberculosis, and anthrax, respectively $[10-12,19,20]$.

The risks associated with FLA infection in Uganda are not known, the reason why this study is being carried out. The associations are expected to be high because significant portions of these communities are using natural environmental water and domestic tap water sources without any definite measures for protecting water systems in QEPA and KLA from these organisms [5]. FLA are not often mentioned as possible infectious agents, yet they have severe pathogenic effects $[25,26]$. Disease effects associated with pathogenic FLA in humans and animals often go undetected because there is scarce information about their distribution. In Uganda, the gap being addressed is that of studying pathogenic FLA in environmental water and domestic tap water systems. This information will help explain the risks associated with water-related illnesses among rural and urban dwelling Ugandans.

\section{Methods}

2.1. Study Areas. The study was conducted in a rural area, Queen Elizabeth Protected Area (QEPA) in western Uganda, and an urban area, Kampala (KLA) City, Uganda. The QEPA is located at $0012 \mathrm{~S}, 3000 \mathrm{E}$ (latitude: 0.2000; longitude: 30.0000 ) and is $1,978 \mathrm{sq}$. $\mathrm{km}$ in size. The protected area is known for its wildlife, including Cape buffaloes (Syncerus caffer caffer), Hippopotami (Hippopotamus amphibius), Nile Crocodiles (Crocodylus niloticus), elephants
(Loxodonta africana), leopards (Panthera pardus), lions (Panthera leo), chimpanzees (Pan troglodytes), and Uganda kobs (Kobus kob thomasi). It is a home to 95 species of mammals and more than 500 species of birds. The protected area is also famous for its volcanic features, including volcanic cones and deep craters. QEPA is a UNESCO "Man and Biosphere Reserve" with 11 village enclaves, all with a fast growing population of humans whose main economic activities are fishing and livestock production. The diversity of animals in the protected area coupled with adjacent human communities makes it a vital hub for sharing of infections at water point sources.

Kampala is the capital city of Uganda lying at latitude $0^{\circ} 18^{\prime} 58^{\prime \prime} \mathrm{N}$, longitude $32^{\circ} 34^{\prime} 55^{\prime \prime} \mathrm{E}$, with $72.97 \mathrm{mi}^{2}\left(189 \mathrm{~km}^{2}\right)$ and divided into five boroughs each consisting of a concentration of slums with a rapidly growing population. Currently estimated at 1,659,600 [27] Kampala's population is increasing steadily, yet service provision is not improving. Water resources used in the city vary from piped water, protected springs, unprotected springs, and natural surface water (lakes, rivers, streams, swamps, roadside gutters, and pasture puddles) which are often relatively unsafe for human consumption.

2.2. Ethical Consideration. This study does not require an ethical statement.

2.3. Study Design. The study involved one year of field work and one year of confirmatory laboratory phase. Laboratory phase involved analysing water samples for FLA. The water sources considered were those from natural environmental and domestic tap water sources. The sampling sites were purposively selected based on their benefit, convenience, and importance to public health.

In QEPA, selection of the study area was based on certain landmarks that included the following: (1) along the Kyambura River, (2) Kazinga Channel banks, (3) Kazinga Midchannel, (4) fish landing sites (FLS), and (5) community piped tap water. In Kampala, the location of sampling sites was based on previous waterborne disease outbreak occurrences. We selected areas in central Kampala (Banda, Kisenyi, Katanga, Kasubi, Kazo, Bwaise, Lubigi, and Makerere) that were reported to have undergone disease outbreak in the last 5 years and collected samples from piped tap water and natural environmental surface water (swamp and stream).

\subsection{Laboratory Methods}

2.4.1. Xenic Cultivation of FLA from Water Samples. We used nonnutritive medium (Page Amoeba Saline solution of $2.5 \mathrm{mM} \mathrm{NaCl}, 1 \mathrm{mM} \mathrm{KH} \mathrm{PO}_{4}, 0.5 \mathrm{mM} \mathrm{Na} \mathrm{HPO}_{4}, 40 \mathrm{mM}$ $\mathrm{CaCl}_{2}$, and $20 \mathrm{mM} \mathrm{MgSO}_{4}$ ) seeded with $0.1 \mathrm{~mL}$ of a heat inactivated 48-hour culture of Escherichia coli BL2 [5, 28]. Fresh water samples in the $50 \mathrm{~mL}$ tubes were centrifuged at $1000 \times \mathrm{g}$ for 15 minutes and supernatant was poured off to expose the pellets. Using sterile Pasteur pipettes, the pellets were removed from all the tubes and each was carefully spread/plated on preseeded NNA-EI agar plates. This was 
followed by incubating the plates at $32^{\circ} \mathrm{C}$ overnight, after which each plate was sealed with a plastic film and incubated upside down at $32^{\circ} \mathrm{C}$ up to 7 days. Three days later, the plates were monitored for detection of amoebae trophozoites until day 7 using an inverted microscope (Motic ${ }^{\circledR}$ AE2000 Binocular, TED PELLA Inc. USA).

2.4.2. DNA Extraction. All DNA was extracted from culture positive plates by chemical lysis and purification [29]. Five hundred microliters of STE buffer $(0.1 \mathrm{M} \mathrm{NaCl}, 1 \mathrm{mM}$ EDTA, $10 \mathrm{mM}$ trischloride, $\mathrm{PH} 8$, and $1 \% \mathrm{SDS}$ ) and $10 \mu \mathrm{L}$ proteinase $\mathrm{K}(10 \mathrm{mg} / \mathrm{mL})$ were added to each sample in an Eppendorf tube. All samples were put in a water bath and incubated at $56^{\circ} \mathrm{C}$ for one hour and then left to cool down before phenol extraction. Phenol chloroform $(521 \mu \mathrm{L})$ was added to each sample, vortexed, and centrifuged at maximum speed $(13200 \mathrm{rpm})$ for 5 minutes. The aqueous layer was transferred to a new Eppendorf tube and the step redone to make 2 phenol-chloroform extractions. The aqueous layer was subjected to another chloroform extraction, centrifuged, and transferred to a new Eppendorf tube after which $1000 \mu \mathrm{L}$ of absolute alcohol (96-100\%) was added to each sample. The samples were put in a freezer at $-80^{\circ} \mathrm{C}$ overnight for precipitation. The following day, all samples were centrifuged at $13200 \mathrm{rpm}$ for 30 minutes and alcohol was poured off. The pellet was washed with $1000 \mu \mathrm{L}$ of $70 \%$ alcohol and centrifuged at $13200 \mathrm{rpm}$ for 15 minutes. Alcohol was poured off to expose the pellet, which was air-dried and dissolved in $50 \mu \mathrm{L}$ of TE buffer.

2.4.3. DNA Amplification. Amplification of $18 \mathrm{~S}$ ribosomal DNA (18S rDNA) from Acanthamoeba and other FLA was done by primer pairs JDP1/JDP2 and CRN5/1137 [12, 30, 31]. Forward primer JDP1 (5'GGCCCAGATCGTTTACCGTGAA- $\left.3^{\prime}\right)$ and reverse primer JDP2 (5'TCTCACAAGCTGCTAGGGAGTCA- ${ }^{\prime}$ ) were genus-specific for Acanthamoeba spp. Forward primer CRN5 (5'CTGGTTGATCCTGCCAGTAG- $\left.3^{\prime}\right)$ and reverse primer 1137 ( $5^{\prime}$ GTGCCCTTCCGTCAAT $-3^{\prime}$ ) obtained amplimers from any eukaryote aiding amplification of the $18 \mathrm{~S}$ ribosomal DNA gene from different FLA.

The reactions were carried out with a DreamTaq PCR kit (Thermo Scientific DreamTaq, USA). A $25 \mu$ L reaction volume containing $12.5 \mu \mathrm{L}$ DreamTaq Green PCR Master Mix (2x), $0.5 \mu \mathrm{M}$ forward primer, $0.5 \mu \mathrm{M}$ reverse primer, $9 \mu \mathrm{L}$ nuclease-free water, and $2.5 \mu \mathrm{L}$ DNA template ( $50 \mathrm{pg}$ concentration) was used. The following conditions were considered for the PCR: initial denaturation at $94^{\circ} \mathrm{C}$ for 3 minutes, followed by 35 cycles with denaturation at $94^{\circ} \mathrm{C}$ for 30 seconds, followed by annealing at $55^{\circ} \mathrm{C}$ for 30 seconds, then extension at $72^{\circ} \mathrm{C}$ for 30 seconds, and a final extension at $72^{\circ} \mathrm{C}$ for 5 minutes. Five microliters of each PCR reaction was tested for successful amplification using agarose gel $(2.5 \% \mathrm{~W} / \mathrm{V})$ stained with ethidium bromide, run against $1 \mathrm{kbp}$ DNA ladder. Once enough electrophoretic separation was reached the agarose gel was observed under a UV gel documentation system, thereafter capturing the gel images.

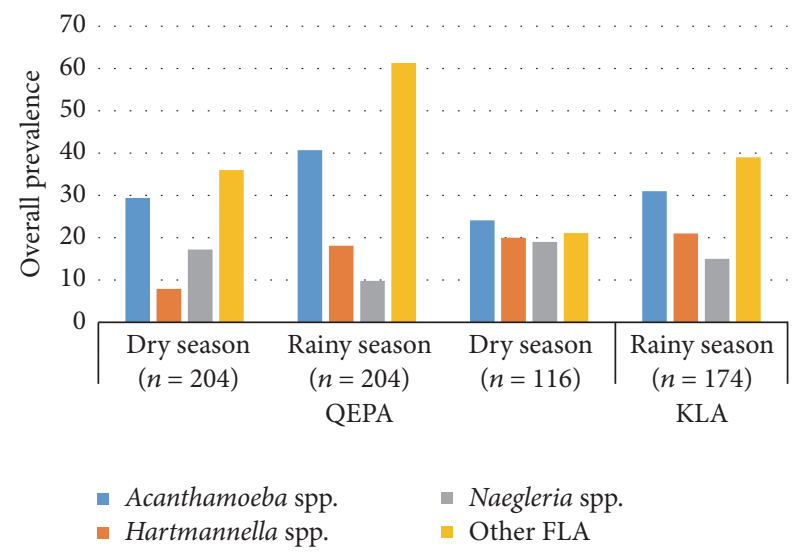

FIGURE 1: Overall seasonal prevalence of FLA.

2.5. Nucleic Acid Sequencing and Analysis. Positive gel samples were extracted and the DNA purified with QIAquick Gel Extraction Kit (Qiagen Inc., Netherlands). Partial 18S rDNA segment of the amoeba isolates was exposed to cycle sequencing with JDP1/JDP2 and CRN5/1137 as sequencing primers [31]. Base trimming of the sequence files to obtain good quality was done by "SeqBuilder" software (Dnastar, USA) and a search for homologues in the NCBI database was done using "blastn" tool. The resultant homologues with query coverage $>70 \%$, identity $>70 \%$, and low $E$ values $(<0)$ were considered. Phylogenetic analysis was done and a dendrogram was constructed [32].

2.6. Statistical Analysis. Data was entered into Excel, from which it was extracted and analysed using SPSS (IBM, USA). Variables were summarised by the use of mean and standard error of the mean (SEM). Application of univariate analysis to compare prevalence across sampling sites was executed using cross-tabulation with a $\chi^{2}$ test. All variables with a $p$ value of $\leq 0.05$ were considered significant. We employed Pearson correlation coefficient $(r)$ to carry out linear correlation analysis between natural/tap water variables and water parasite presence.

\section{Results}

\subsection{Prevalence of Parasites}

3.1.1. Overall Seasonal Prevalence. The water samples were collected during cold rainy (November, March, and July) and cool dry (January, May, and September) seasons. Overall, FLA parasite prevalence in both study sites was higher during the rainy season except for Naegleria spp. that was higher in the dry season in QEPA (Figure 1).

3.1.2. Overall Prevalence and Mean. The prevalence and mean (SEM) of the parasites from different sources are shown in Table 1. Both natural environmental and domestic tap water sources were contaminated with FLA. The overall prevalence of Acanthamoeba spp. and other FLA in QEPA and Kampala was as follows: Acanthamoeba spp. (QEPA, 
TABLE 1: The overall prevalence of the FLA.

\begin{tabular}{|c|c|c|c|c|c|c|}
\hline \multirow{2}{*}{ Parasite } & \multicolumn{3}{|c|}{ QEPA } & \multicolumn{3}{|c|}{ KLA } \\
\hline & Freq $(n=408)$ & Prev (\%) & Mean \pm SE & Freq $(n=290)$ & Prev (\%) & Mean \pm SE \\
\hline Acanthamoeba spp. & 143 & 35 & $1.9 \pm 0.2$ & 82 & 28.3 & $2.8 \pm 0.5$ \\
\hline Naegleria spp. & 55 & 13.5 & $0.4 \pm 0.1$ & 48 & 16.6 & $1.4 \pm 0.3$ \\
\hline Hartmannella spp. & 77 & 18.9 & $1.2 \pm 0.1$ & 67 & 23.1 & $1.4 \pm 0.3$ \\
\hline
\end{tabular}

Freq: frequency. Prev: prevalence.

TABLE 2: Prevalence and mean across water sources.

\begin{tabular}{|c|c|c|c|c|}
\hline \multirow{2}{*}{ Parasite } & \multicolumn{2}{|c|}{ QEPA } & \multicolumn{2}{|c|}{ KLA } \\
\hline & Tap water $(n=84)$ & Environmental water $(n=324)$ & Tap water $(n=170)$ & Environmental water $(n=65)$ \\
\hline \multicolumn{5}{|c|}{ Acanthamoeba spp. } \\
\hline$(+)(\%)$ & $36(43)$ & $107(33)$ & $48(28.2)$ & $15(23.1)$ \\
\hline Mean \pm SEM & $2.26 \pm 0.4$ & $8.92 \pm 1.6$ & $5.2 \pm 1.2$ & $2.4 \pm 1.3$ \\
\hline \multicolumn{5}{|c|}{ Hartmannella spp. } \\
\hline$(+)(\%)$ & $19(22.6)$ & $58(17.9)$ & $38(22.4)$ & $10(15.4)$ \\
\hline Mean \pm SEM & $1.20 \pm 0.15$ & $4.93 \pm 0.92$ & $4.4 \pm 1.0$ & $0.9 \pm 0.16$ \\
\hline \multicolumn{5}{|l|}{ Naegleria spp. } \\
\hline$(+)(\%)$ & $12(14.3)$ & $43(13.3)$ & $25(14.7)$ & $9(13.8)$ \\
\hline Mean \pm SEM & $0.5 \pm 0.15$ & $1.58 \pm 0.6$ & $3.5 \pm 1.1$ & $0.5 \pm 0.3$ \\
\hline
\end{tabular}

35\%; KLA, 28.3\%), Hartmannella spp. (QEPA, 18.9\%; KLA, 23.1\%), and Naegleria spp. (QEPA, 13.5\%, KLA, 16.6\%). The mean $( \pm$ SEM) was highest for Acanthamoeba spp., followed by Hartmannella spp. and, lastly, Naegleria spp.

3.1.3. Prevalence of Parasites across Sampling Sites. Prevalence of parasites across water source (natural and tap water) considered is presented in Table 2. Acanthamoeba spp. were the most prevalent parasite across all sources. Natural environmental water had significantly higher mean values compared to tap water in both study sites.

3.1.4. Molecular Identification and Phylogenetic Analysis. Following FLA sequencing, the products were blasted and compared with the GenBank results from NCBI (Figure 2; Table 3). Comparisons between the FLA isolated in Uganda and those from the NCBI database were made and differences in divergence noted (Figure 2; Table 4) were assessed. The species identified were Acanthamoeba spp. (various T-genotypes), Acanthamoeba polyphaga, Hartmannella vermiformis, Nuclearia pattersoni, Echinamoeba exundans, Bodomorpha minima, and Cercomonas agilis. The Acanthamoeba sequences got belonged to the group of sequence types T1, T2, T4, and T11 (Table 3).

The confirmed Acanthamoeba genotype T1 was isolated from tap water in Bwaise, whereas T2 and T11 were isolated from the Kazinga Channel water in QEPA. Genotype T4 which is usually the commonest Acanthamoeba T-genotype was isolated from tap water in Katunguru Trading Centre, Kasaka landing site, Albertine Restaurant in QEPA, and
Kisenyi slum in Kampala. The Hartmannella vermiformis confirmed was isolated from fish landing sites in QEPA. The nonpathogenic FLA (Table 3) were isolated from a variety of tap water samples in Albertine Restaurant, Thembo Restaurant, and Katunguru Trading Center, as well as natural surface water from fish landing sites and the Kyambura River in QEPA. In Kampala natural water samples that were positive for FLA were from Lubigi swamps.

All the parasites identified in this study were matched with the reported diseases they cause in humans (Table 3 ).

\section{Discussion}

We investigated the presence of FLA in natural and domestic (tap) water in QEPA and KLA. The prevalence and mean $( \pm$ SEM) of Acanthamoeba spp. in all cases were higher than Naegleria and Hartmannella spp. All FLA were more prevalent in the rainy season except Naegleria spp. that were higher in the dry season. Most waterborne parasites increase in number during the rainy season due to contamination of the water sources with sewage, soil, and other organic matters from water run-off $[5,33]$. Tap water had a higher prevalence of FLA than the natural water source. There is not much data in Uganda to compare with the present findings but studies from other countries [34, 35] also documented a higher frequency of Acanthamoeba compared to other FLA in environmental and tap water samples in alkaline water at the same temperatures. The first ever study of FLA in Uganda documented higher prevalence and mean of Acanthamoeba than other FLA in both environmental and tap water [5]. 


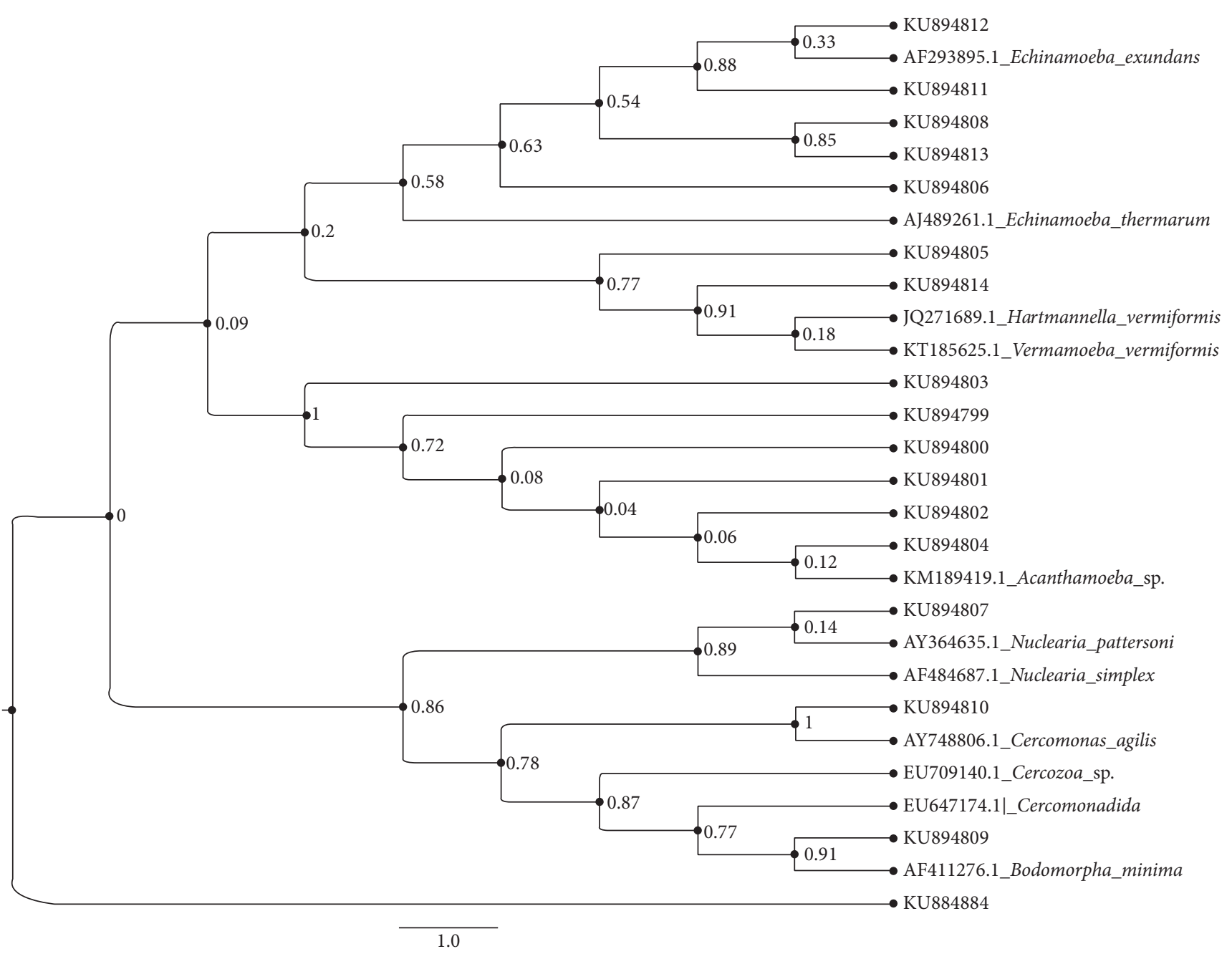

FIGURE 2: Phylogenetic tree based on neighbor-joining, showing the divergence of FLA. Comparison with closely related species from the GenBank database sequences with their accession numbers (GenBank NCBI).

Acanthamoeba spp. are more commonly encountered probably because they are more involved in a predator-prey relationship with microbial colonies. The high numbers could be explained by the presence of organic matter from rotting leaves, animal, and human faeces which are from the run-of $\mathrm{f}$ from the land that often concentrate at the banks of the water bodies. This is known to exacerbate microbial biofilm formation and as a result facilitate the proliferation of FLA. It is believed that there are more FLA when there is an accumulation of more organic matter in soil and water [36, 37]. Previous studies report that microorganisms settle on the inner surfaces of water pipes later becoming a source of secondary microbial contamination [38].

The primer pair JDP1/JDP2 that was used is more specific in the amplification of Acanthamoeba DNA [30, 39], whereas CRN5/1137 amplifies any eukaryote DNA [30]. Acanthamoeba sequence types can be grouped T1 to T20 [40]. Blasting of sequences and comparison with those from NCBI database produced 7 Acanthamoeba, 5 Echinamoeba, 2 Hartmannella, 1 Bodomorpha, 1 Nuclearia, and 1 Cercomonas partial sequences. Acanthamoeba genotypes T1, T2, and T4 were mainly isolated from tap water samples, whereas 11 were isolated from environmental water samples. This is in agreement with previous studies that indicate that T2 Acanthamoeba genotype is mainly found in the environment and is phylogenetically related to T6 and they have also been both isolated from clinical AK cases in humans [40]. Acanthamoeba genotype T11 is closely related to T4 and has been found to also cause AK [33]. Acanthamoeba of T4 genotype is reported as the most commonly encountered T-genotype group in both environmental water and clinical samples and also the most diverse [33]. Genotype T1 is notorious for granulomatous amoebic encephalitis [11] whereas others may cause keratitis, cutaneous infections, and sinusitis in humans [18, 41]. Hartmannella vermiformis originally thought to be nonpathogenic [42] has over the past decade been repeatedly reported in a number of mixed human AK infections $[4,13$, 24]. Although other FLA isolated in this study are considered nonpathogenic, it is possible that they too could become virulent anytime, given a conducive environment.

Infective trophozoites of FLA in the environment are maintained and spread by water during rainy seasons when 
TABLE 3: Isolated free-living amoeba, accession numbers, water source, and associated diseases in humans.

\begin{tabular}{|c|c|c|c|c|}
\hline Accession & Source & FLA & Diseases & Characteristics \\
\hline KU884884 & Tap KLA Bwaise & Acanthamoeba spp. (T1) & Encephalitis [11, 21] & $\begin{array}{l}\text { Mental status changes, } \\
\text { hemiparesis, meningismus, and } \\
\text { ataxia }[22]\end{array}$ \\
\hline KU894799 & Tap QEPA Katunguru & Acanthamoeba spp. (T2) & Keratitis $[11,21]$ & $\begin{array}{l}\text { Blinding infection of the cornea } \\
{[22,23]}\end{array}$ \\
\hline KU894800 & Tap QEPA Katunguru & Acanthamoeba spp. (T4) & Keratitis $[11,21]$ & $\begin{array}{l}\text { Blinding infection of the cornea } \\
{[22,23]}\end{array}$ \\
\hline KU894801 & Tap QEPA Albertine & $\begin{array}{c}\text { Acanthamoeba polyphaga } \\
\text { (T4) }\end{array}$ & Keratitis $[11,21]$ & $\begin{array}{l}\text { Blinding infection of the cornea } \\
{[22,23]}\end{array}$ \\
\hline KU894802 & Tap QEPA Kasaka & Acanthamoeba spp. (T4) & Keratitis $[11,21]$ & $\begin{array}{l}\text { Blinding infection of the cornea } \\
{[22,23]}\end{array}$ \\
\hline KU894803 & Tap KLA Kisenyi & Acanthamoeba spp. (T4) & Keratitis $[11,21]$ & $\begin{array}{l}\text { Blinding infection of the cornea } \\
{[22,23]}\end{array}$ \\
\hline KU894804 & $K C B Q E P A$ & Acanthamoeba spp. (T11) & $\begin{array}{c}\text { Keratitis and } \\
\text { encephalitis [22] }\end{array}$ & $\begin{array}{l}\text { Disseminated disease, blindness, } \\
\text { and CNS function impairment } \\
{[22]}\end{array}$ \\
\hline KU894805 & FLS QEPA & Hartmannella vermiformis & Keratitis $[4,13,24]$ & $\begin{array}{l}\text { Blinding infection of the cornea } \\
\text { [22] }\end{array}$ \\
\hline KU894806 & TAP QEPA Albertine & Echinamoeba exundans & Unknown & - \\
\hline KU894807 & TAP QEPA Thembo & Nuclearia pattersoni & Unknown & - \\
\hline KU894808 & TAP QEPA Katunguru & Echinamoeba exundans & Unknown & - \\
\hline KU894809 & FLS QEPA & Bodomorpha minima & Unknown & - \\
\hline KU894810 & Lubigi KLA & Cercomonas agilis & Unknown & - \\
\hline KU894811 & R. Kyambura QEPA & Echinamoeba exundans & Unknown & - \\
\hline KU894812 & R. Kyambura QEPA & Echinamoeba exundans & Unknown & - \\
\hline KU894813 & TAP QEPA Albertine & Echinamoeba exundans & Unknown & - \\
\hline KU894814 & TAP QEPA Thembo & Hartmannella vermiformis & Keratitis $[4,13,24]$ & $\begin{array}{l}\text { Blinding infection of the cornea } \\
\text { [22] }\end{array}$ \\
\hline
\end{tabular}

FLS: fish landing sites; KCB: Kazinga Channel banks; KLA: Kampala; QEPA: Queen Elizabeth Protected Area. R. Kyambura: the Kyambura River.

there is a run-off of water containing human and animal faecal matter from land into the water bodies, which eventually end up at the points where communities fetch and utilise the water. Most FLA prevalence and mean intensities are higher in the rainy season than dry season [43]. However, parasite incidences can be high throughout the rainy and dry seasons, often indicating poor disposal of human and animal excreta and continuous patterns of infection [43]. In a natural water environment, pathogens have been isolated widely from many water sources used by rural dwelling households [33, $44,45]$. Often inadequately treated domestic water (drinking, bathing, cooking, and recreational water) has an abundance of such pathogens. Water bodies are usually contaminated by high concentrations organisms from agricultural run-off, urban wastewater effluents [46], and for the case of QEPA and KLA human and animal faecal contamination. The QEPA and KLA local communities have few poorly built latrines, most of which are already filled up, compelling many to dig small holes in the ground and defecate outside, on open land. When there is a heavy downpour of rain, human, and animal the faecal material is washed off into the public water supply system. Upon using this water, exposure to a variety of protozoan parasites is highly likely. The risk of human infection is much higher in children and immunocompromised individuals such as those who have HIV/AIDS, diabetes, and cancer and those who have recently undergone organ transport [3].

\section{Conclusion}

The findings from the present study indicate that there is reasonably high contamination of both natural and domestic water systems with Acanthamoeba spp., Hartmannella spp., Naegleria spp., and other FLA. This is evidence that the water being used is of poor quality and predisposes communities to infectious agents. With the fact that there is proof that some are pathogenic and can be vectors of many emerging/reemerging infectious agents, it is imperative to prevent them from contaminating domestic water sources.

\section{Abbreviations}

${ }^{\circ} \mathrm{C}: \quad$ Celsius

AK: Amoebic keratitis

CNS: Central nervous system

Environ: Environment 


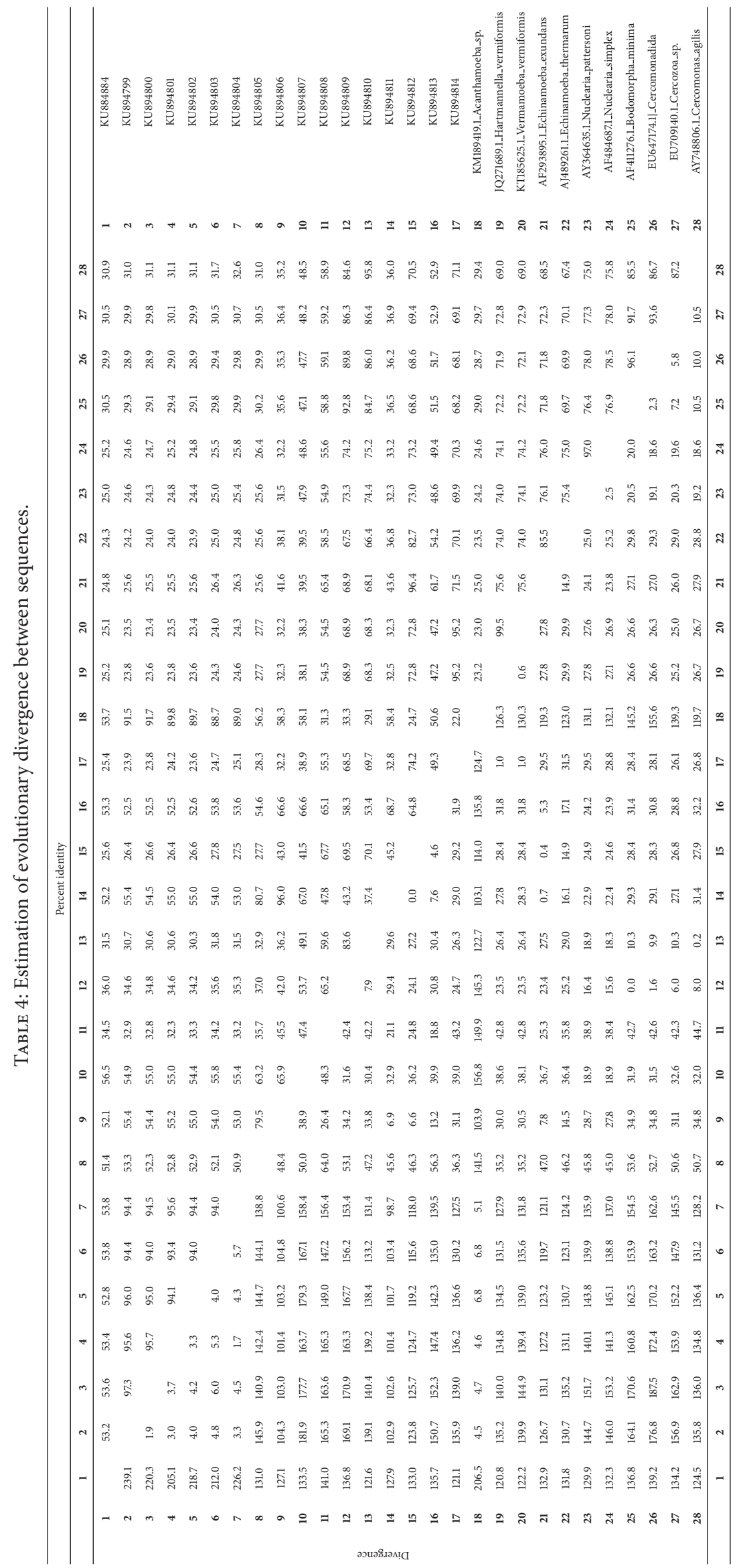




$\begin{array}{ll}\text { FLA: } & \text { Free-living amoeba } \\ \text { FLS: } & \text { Fish landing site } \\ \text { G: } & \text { Gravitational force } \\ \text { g: } & \text { Grams } \\ \text { GAE: } & \text { Granulomatous amoebic encephalitis } \\ \text { HIV/AIDS: } & \text { Human immunodeficiency } \\ & \text { virus/acquired immune deficiency } \\ & \text { syndrome } \\ \text { KCB: } & \text { Kazinga Channel bank } \\ \text { KCM: } & \text { Kazinga Channel middle } \\ \text { KLA: } & \text { Kampala } \\ \text { L: } & \text { Liter } \\ \text { mg: } & \text { Milligrams } \\ \text { mL: } & \text { Milliliters } \\ \text { mM: } & \text { Millimolar } \\ \text { NCBI: } & \text { National Center for Biotechnology } \\ & \text { Information } \\ \text { No.: } & \text { Number } \\ \text { PA: } & \text { Protected area } \\ \text { PAM: } & \text { Primary amoebic } \\ \text { pg: } & \text { meningoencephalitis } \\ \text { pmol: } & \text { Picogram } \\ \text { Prev: } & \text { Picomoles } \\ \text { QEPA: } & \text { Prevalence } \\ \text { rpm: } & \text { Queen Elizabeth Protected Area } \\ \text { SG: } & \text { Revolutions per minute } \\ \text { UNESCO: } & \text { Specific gravity } \\ \text { Un-ID Amoeba: } & \text { United Nations Educational, } \\ \text { V: } & \text { Volts } \\ \mu S: & \text { Microsecontific and Cultural Organization } \\ & \end{array}$

\section{Competing Interests}

There are no competing interests.

\section{References}

[1] A. J. Martinez and G. S. Visvesvara, "Free-living, amphizoic and opportunistic amebas," Brain Pathology, vol. 7, no. 1, pp. 583598, 1997.

[2] WHO, Combating Waterborne Disease at the Household Level/International Network to Promote Household Water Treatment and Safe Storage, World Health Organization, Geneva, Switzerland, 2007.

[3] CDC, Domestic Water, Sanitation, and Hygiene Epidemiology, Center for Disease Control, Atlanta, Ga, USA, 2015, http://www.cdc.gov/ncezid/dfwed/waterborne/domestic.html.

[4] H. Abedkhojasteh, M. Niyyati, F. Rahimi, M. Hei-Dari, S. Farnia, and M. Rezaeian, "First report of Hartmannella keratitis in a cosmetic soft contact lens wearer in Iran," Iranian Journal of Parasitology, vol. 8, no. 3, pp. 481-485, 2013.

[5] C. Sente, J. Erume, I. Naigaga et al., "Occurrence and genetic characterisation of Acanthamoeba spp. from environmental and domestic water sources in Queen Elizabeth Protected Area, Uganda," Parasites \& Vectors, vol. 9, article 127, 2016.

[6] J. De Jonckheere, "Naegleria australiensis sp. nov., another pathogenic Naegleria from water," Protistologica, vol. 17, pp. 423-429, 1981.
[7] J. F. De Jonckheere, "Isoenzyme patterns of pathogenic and nonpathogenic Naegleria spp. using agarose isoelectric focusing," Annales de Microbiologie, vol. 133, no. 2, pp. 319-342, 1982.

[8] J. F. De Jonckheere, "A century of research on the amoeboflagellate genus Naegleria," Acta Protozoologica, vol. 41, no. 4, pp. 309-342, 2002.

[9] CDC, "Acanthamoeba keratitis associated with contact lensesUnited States," Morbidity and Mortality Weekly Report, vol. 35, no. 25, pp. 405-408, 1986.

[10] V. A. Dunand, S. M. Hammer, R. Rossi et al., "Parasitic sinusitis and otitis in patients infected with human immunodeficiency virus: report of five cases and review," Clinical Infectious Diseases, vol. 25, no. 2, pp. 267-272, 1997.

[11] S. Amir, Acanthamoeba castellanii as a Host and Model to Study Bacterial Virulence, Karolinska Universitetssjukhuset, Huddinge, Sweden, 2009.

[12] R. Dey, P. S. Hoffman, and I. J. Glomski, "Germination and amplification of anthrax spores by soil-dwelling amoebas," Applied and Environmental Microbiology, vol. 78, no. 22, pp. 8075-8081, 2012.

[13] R. Solgi, M. Niyyati, A. Haghighi, and E. N. Mojarad, "Occurrence of thermotolerant Hartmannella vermiformis and Naegleria spp. in hot springs of Ardebil Province, Northwest Iran," Iranian Journal of Parasitology, vol. 7, no. 2, pp. 47-52, 2012.

[14] Uganda: Cholera, WHO Regional Office for Africa, 2012, http:// www.afro.who.int/en/clusters-a-programmes/dpc/epidemic-a -pandemic-alert-and-response/3601-uganda-cholera-situation -as-of-31-march-2012.html.

[15] G. Ojore, Uganda: 2,000 Children Die of Diarrhoea in Africa Daily-WaterAid, New Vision Publishing, Kampala, Uganda, 2012.

[16] Uganda rural water services. Inadequate quantity, low quality, satisfied users, IRC, 2014, http://www.ircwash.org/news/uganda -rural-water-services-inadequate-quantity-low-quality-satisfied -users.

[17] WHO Regional Office for Africa, Emergencies Preparedness, and Response to Typhoid Fever, Uganda, 2015, http://www.who .int/csr/don/17-march-2015-uganda/en/.

[18] Naegleria Infection, Medscape Drugs \& Diseases, 2015, http:// emedicine.medscape.com/article/223910-overview\#a5.

[19] G. S. Visvesvara, H. Moura, and F. L. Schuster, "Pathogenic and opportunistic free-living amoebae: Acanthamoeba spp., Balamuthia mandrillaris, Naegleria fowleri, and Sappinia diploidea," FEMS Immunology and Medical Microbiology, vol. 50, no. 1, pp. $1-26,2007$.

[20] L. J. Stockman, C. J. Wright, G. S. Visvesvara, B. S. Fields, and M. J. Beach, "Prevalence of Acanthamoeba spp. and other freeliving amoebae in household water, Ohio, USA-1990-1992," Parasitology Research, vol. 108, no. 3, pp. 621-627, 2011.

[21] G. S. Visvesvara, "Amebic meningoencephalitides and keratitis: challenges in diagnosis and treatment," Current Opinion in Infectious Diseases, vol. 23, no. 6, pp. 590-594, 2010.

[22] N. Crum-Cianflone, Acanthamoeba, Naval Medical Center at San Diego, San Diego, Calif, USA, 2015.

[23] F. Marciano-Cabral and G. A. Cabral, "Acanthamoeba spp. as agents of disease in humans," Clinical Microbiology Reviews, vol. 16, no. 2, pp. 273-307, 2003.

[24] J. Lorenzo-Morales, E. Martínez-Carretero, N. Batista et al., "Early diagnosis of amoebic keratitis due to a mixed infection with Acanthamoeba and Hartmannella," Parasitology Research, vol. 102, no. 1, pp. 167-169, 2007. 
[25] Z. Szénási, T. Endo, K. Yagita, and E. Nagy, "Isolation, identification and increasing importance of 'free-living' amoebae causing human disease," Journal of Medical Microbiology, vol. 47, no. 1, pp. 5-16, 1998.

[26] H. Trabelsi, F. Dendana, A. Sellami et al., "Pathogenic freeliving amoebae: epidemiology and clinical review," Pathologie Biologie, vol. 60, no. 6, pp. 399-405, 2012.

[27] Uganda Bureau of Statistics, Estimated Population of Kampala in 2002, 2010 \& 2011, Uganda Bureau of Statistics, 2014.

[28] F. L. Schuster and G. S. Visvesvara, "Free-living amoebae as opportunistic and non-opportunistic pathogens of humans and animals," International Journal for Parasitology, vol. 34, no. 9, pp. 1001-1027, 2004.

[29] M. Pélandakis and P. Pernin, "Use of multiplex PCR and PCR restriction enzyme analysis for detection and exploration of the variability in the free-living amoeba Naegleria in the environment," Applied and Environmental Microbiology, vol. 68, no. 4, pp. 2061-2065, 2002.

[30] J. M. Schroeder, G. C. Booton, J. Hay et al., "Use of subgenic 18S ribosomal DNA PCR and sequencing for genus and genotype identification of Acanthamoebae from humans with keratitis and from sewage sludge," Journal of Clinical Microbiology, vol. 39, no. 5, pp. 1903-1911, 2001.

[31] G. C. Booton, D. J. Kelly, Y.-W. Chu et al., "18S ribosomal DNA typing and tracking of Acanthamoeba species isolates from corneal scrape specimens, contact lenses, lens cases, and home water supplies of Acanthamoeba keratitis patients in Hong Kong," Journal of Clinical Microbiology, vol. 40, no. 5, pp. 16211625, 2002.

[32] K. Tamura, G. Stecher, D. Peterson, A. Filipski, and S. Kumar, "MEGA6: molecular evolutionary genetics analysis version 6.0," Molecular Biology and Evolution, vol. 30, no. 12, pp. 2725-2729, 2013.

[33] P. Bonilla-Lemus, A. S. Caballero Villegas, J. Carmona Jiménez, and A. Lugo Vázquez, "Occurrence of free-living amoebae in streams of the Mexico Basin," Experimental Parasitology, vol. 145, supplement, pp. S28-S33, 2014.

[34] S. Ghadar-Ghadr, K. Solhjoo, M. Norouz-Nejad, R. Rohi, and S. Zia-Jahromi, "Isolation and identification of free living amoeba (Naegleria and Acanthamoeba) in Shiraz water resources by morphological criteria," Pars of Jahrom University of Medical Sciences, vol. 10, no. 3, pp. 33-42, 2012.

[35] S. Ghadar-Ghadr, K. Solhjoo, M. Norouz-Nejad, R. Rohi, and S. Zia-Jahromi, "Isolation and identification of free living amoeba (Naegleria and Acanthamoeba) in Shiraz water resources by morphological criteria," Journal of Jahrom University of Medical Sciences, vol. 10, no. 3, pp. 26-33, 2012.

[36] S. Rodriguez-Zaragoza, E. Mayzlish, and Y. Steinberger, "Vertical distribution of the free-living amoeba population in soil under desert shrubs in the Negev Desert, Israel," Applied and Environmental Microbiology, vol. 71, no. 4, pp. 2053-2060, 2005.

[37] M. Pruden, J. Falkinham, K. Williams, H. Wang, K. Martins, and W. Rhoads, "Relationship between biodegradable organic matter and pathogen concentrations in premise plumbing," Water Research Foundation, pp. 58-90, 2013.

[38] A. Rożej, A. Cydzik-Kwiatkowska, B. Kowalska, and D. Kowalski, "Structure and microbial diversity of biofilms on different pipe materials of a model drinking water distribution systems," World Journal of Microbiology \& Biotechnology, vol. 31, no. 1, pp. 37-47, 2015.

[39] M. H. Vodkin, D. K. Howe, G. S. Visvesvara, and G. L. McLaughlin, "Identification of Acanthamoeba at the generic and specific levels using the polymerase chain reaction," The Journal of Protozoology, vol. 39, no. 3, pp. 378-385, 1992.

[40] M. Crary, Genetic Variability and Its Relationship to Acanthamoeba Pathogenesis, Molecular Genetics, Ohio State University, Columbus, Ohio, USA, 2012.

[41] M. S. Torno Jr., R. Babapour, A. Gurevitch, and M. D. Witt, "Cutaneous acanthamoebiasis in AIDS," Journal of the American Academy of Dermatology, vol. 42, no. 2, part 2, pp. 351-354, 2000.

[42] J. F. De Jonckheere and S. Brown, "There is no evidence that the free-living ameba Hartmannella is a human parasite," Clinical Infectious Diseases, vol. 26, no. 3, p. 773, 1998.

[43] P. W. Woodburn, L. Muhangi, S. Hillier et al., "Risk factors for helminth, malaria, and HIV infection in pregnancy in Entebbe, Uganda," PLoS Neglected Tropical Diseases, vol. 3, no. 6, article e473, 2009.

[44] S. Onichandran, T. Kumar, C. C. Salibay et al., "Waterborne parasites: a current status from the Philippines," Parasites \& Vectors, vol. 7, no. 1, article 244, 2014.

[45] T. Kumar, S. Onichandran, Y. A. L. Lim et al., "Comparative study on waterborne parasites between Malaysia and Thailand: a new insight," The American Journal of Tropical Medicine and Hygiene, vol. 90, no. 4, pp. 682-689, 2014.

[46] D. Carmena, Waterborne Transmission of Cryptosporidium and Giardia: Detection, Surveillance and Implications for Public Health, Faculty of Medicine IC, London, UK, 2010. 

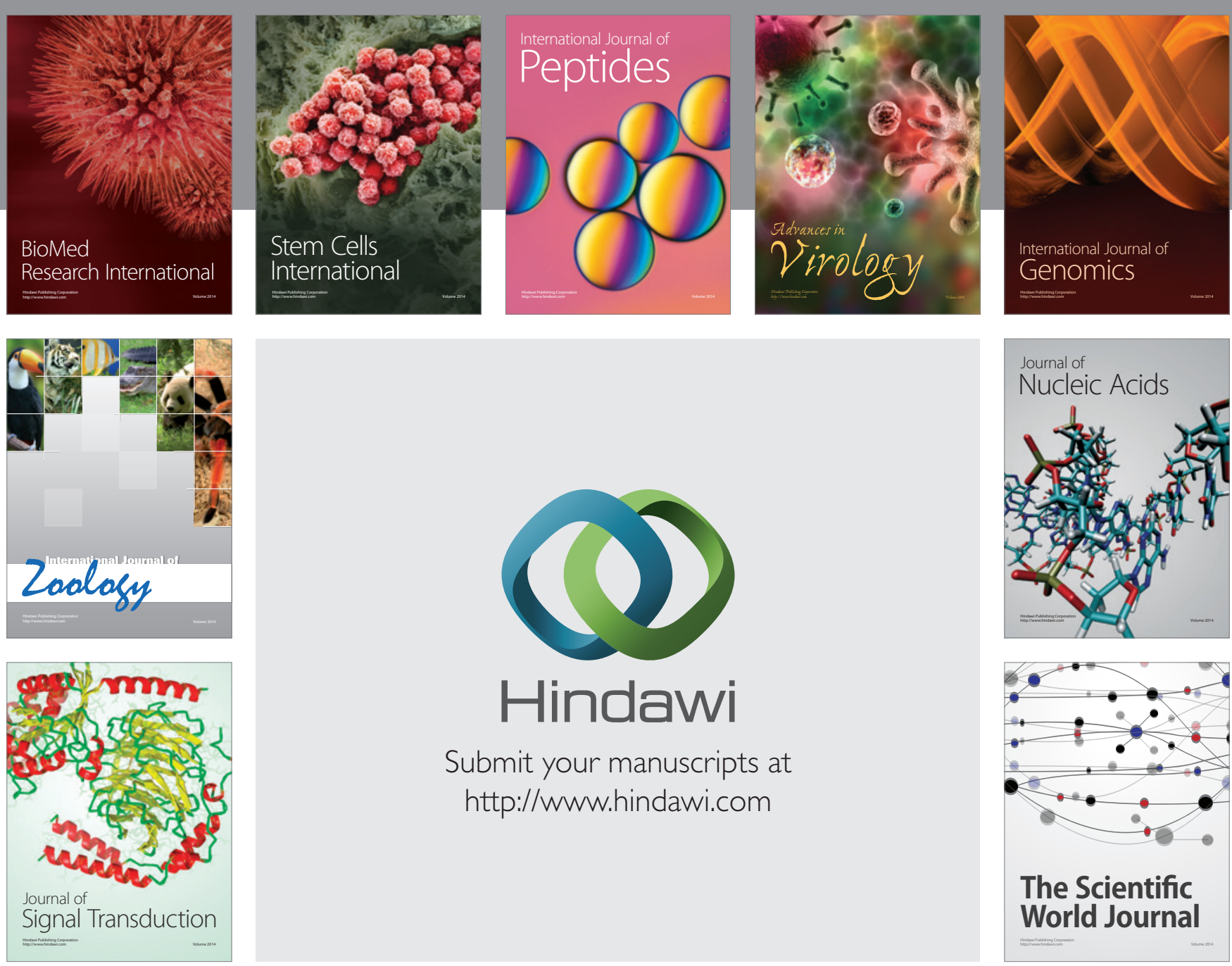

Submit your manuscripts at

http://www.hindawi.com
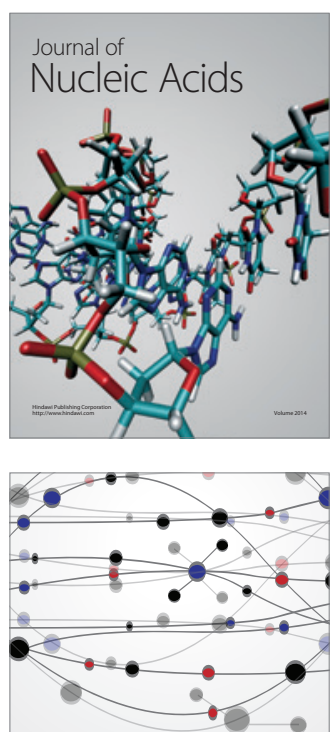

The Scientific World Journal
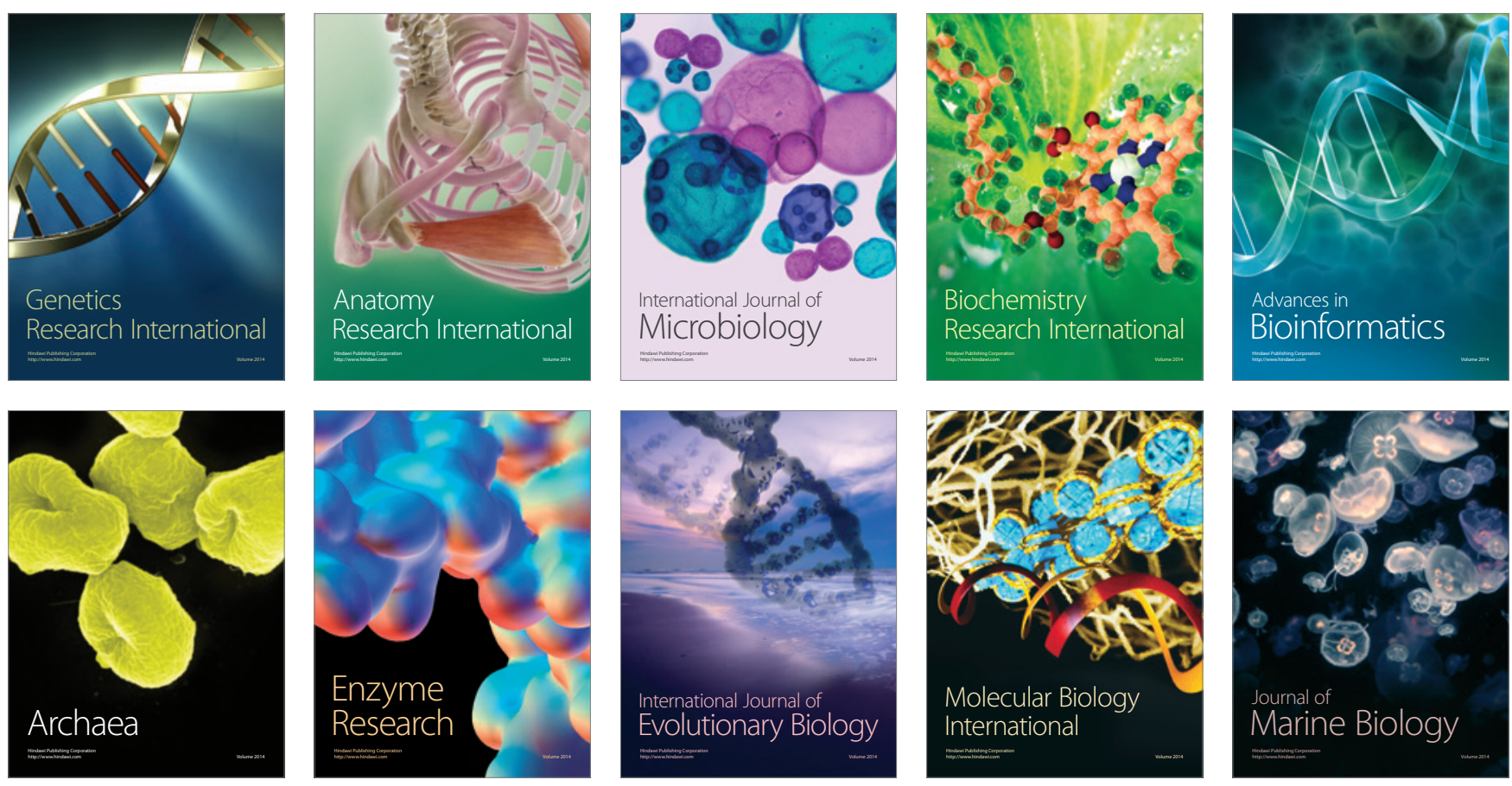